\title{
Prevention and management of dental decay in the pre-school child
}

\section{Scottish Intercollegiate Guideline Network. No. 83. Prevention and management of dental decay in the pre-school child. Edinburgh: Scottish Intercollegiate Guideline Network (SIGN). ISBN $189989344 X 2005$.}

Scope and purpose The guideline addresses effective strategies for preventing and managing dental decay in the preschool child. The nature of dental decay, its diagnosis and its epidemiology are reviewed. Methods for prevention and management of the disease at an individual and population level are reviewed and identification of children at increased risk of developing dental disease is discussed.

Methodology The guideline was synthesised following the SIGN methodology (see www.sign.ac.uk/guidelines/fulltext/50/index.html). Searches were devised in collaboration with members of the guidelinedevelopment group. Searches for guidelines used Embase and Medline for the period 1996-2003 and a range of websites. Searches for systematic reviews, meta-analyses, randomised controlled trials (RCT) and observational studies were carried out with the Cochrane library, Embase and Medline (1990-2003, with a final update in June 2004). The definitions of the types of evidence and the grading of recommendations used in this guideline originate from the US Agency for Health Care Policy and Research ${ }^{1}$ and are set out in Tables 1 and 2. Review and updating The guideline was published in November 2005 and will be considered for updating in 3 years.

Clinical recommendations For summary, see Table 3 below.

Research recommendations During the identification and critical appraisal of evidence for this guideline, it became apparent that there is limited evidence from high-quality, rigorous and methodologically sound research, and that the evidence currently available is often poor and incomplete. The guideline development group recommended that research should be carried out

- evaluating the benefits, and potential risks of fluorosis, of a community-based water fluoridation scheme in the current environment in Scotland,

- determining the relationship between oral health and health inequalities,

- exploring how dental services should be provided in areas of deprivation to minimise inequalities,

- evaluating community-based prevention programmes, including the involvement of health visitors,

- ascertaining the most effective combination of preventive, or preventive and restorative, interventions for different caries-risk groups, with different levels of deprivation, in different settings,

- examining the relationship between age and caries experience in the preschool child,

- describing the caries experience of preschool children with special needs, - completing RCT to investigate the apparent caries-reducing effect of sugar-substituted sweets,

- investigating the effect of breast- and bottle-feeding on the development of early childhood caries in studies that control for other dietary factors,

Address for correspondence: SIGN, 28 Thistle Street, Edinburgh EH2 1EN, UK. www.sign.ac.uk/
Table 1. Statements of evidence

la Evidence obtained from meta-analysis of RCT

Ib Evidence obtained from at least one RCT

lia Evidence obtained from at least one well-designed controlled study without randomisation

lib Evidence obtained from at least one other type of well-designed quasiexperimental study

III Evidence obtained from well-designed nonexperimental descriptive studies, such as comparative studies, correlation studies and case studies

IV Evidence obtained from expert committee reports or opinions and/or clinical experiences of respected authorities

RCT, Randomised controlled trial.

\section{Table 2. Grades of recommendations}

A Requires at least one RCT as part of a body of literature of overall good quality and consistency addressing the specific recommendation (evidence levels $\mathrm{la}, \mathrm{lb}$ )

B Requires the availability of well-conducted clinical studies but no RCT on the topic of recommendation (evidence levels Ila, IIb, III)

C Requires evidence obtained from expert committee reports or opinions and/or clinical experiences of respected authorities.

Indicates an absence of directly applicable clinical studies of good quality (evidence level IV)

GPP Good practice points: recommended best practice based on the clinical experience of the guideline development group

RCT, Randomised controlled trial.

- identifying effective interventions to improve the diet at weaning and preschool age,

- examining the effectiveness of structured nursery toothbrushing programmes,

- determining the optimum age at which to commence brushing children's teeth,

- exploring the effect of one-to-one dental health education on health outcomes,

- evaluating the effectiveness of dental health education programmes promoting the use of fluoride outside a structured programme,

- examining the effects of maternal use of xylitol in preventing caries in young children,

- identifying specific barriers to the implementation of oral health strategies, and how to overcome them,

- assessing the effectiveness of preventive advice delivered by the dental team in the dental practice environment,

- determining the effectiveness of fluoride and chlorhexidine varnishes, and fissure sealing in the primary dentition,

- conducting RCT in primary care to investigate the relative efficacy of conventional restorative management of carious primary teeth including the role of general anaesthetic compared with alternative techniques, and with a prevention-only approach,

- establishing the effect of remuneration on quality of dental care and availability of dental practitioners. 


\section{Table 3. Clinical recommendations}

Diagnosis

GPP

D

As thorough a caries diagnostic examination should be performed as the child's level of cooperation permits

The use of bitewing radiography for caries diagnosis should be considered for preschool children attending for dental care, particularly if they are assessed as being at increased risk of dental caries. The timing of subsequent radiographic examinations should be based on the individual's caries risk status

B Caries should be diagnosed as early as possible to allow management before cavitation and pulpal involvement, and to identify caries-active patients and those at increased risk of caries in the future

C Practitioners should receive training in clinical and radiographic caries diagnosis

Predicting caries risk

C A dental practice-based caries risk assessment should be carried out on individual preschool children and should include the following risk indicators: evidence of previous caries experience; resident in a deprived area; healthcare worker's opinion; oral mutans streptococci counts (if available)

Children whose families live in a deprived area should be considered as at increased risk of early childhood caries when developing preventive programmes

\section{Toothbrushing with fluoride}

A Children should have their teeth brushed with fluoride toothpaste containing 1000 ppm ( $\pm 10 \%)$ fluoride

C Children should have their teeth brushed, or be assisted with toothbrushing by an adult, at least twice a day, with a smear or pea-sized amount of fluoride toothpaste
ootherir teeth brushed, or be assisted with toothbrushi

A $\quad$ Toothbrushing should commence as soon as the primary teeth erupt

A Children's teeth can be brushed with either manual or powered toothbrushes

\section{Diet and nutrition}

B Pregnant women should be advised that there is no benefit to the child of taking fluoride supplements during pregnancy

C Members of the dental team should support and promote breastfeeding according to current recommendations

GPP Parents and carers should be advised that soya infant formulae are potentially cariogenic and should be used only when medically indicated

GPP Parents and carers should be advised not to put children to bed with a bottle or feeder cup

B Parents and carers should be advised that foods and confectionery containing free sugars should be minimised, and if possible restricted to meal times

C Parents and carers should be advised that drinks containing free sugars, including natural fruit juices, should be avoided between meals, and should never be put in a feeding bottle. Water or milk may be given instead

C Parents and carers should be advised that confectionery and beverages containing sugar substitutes are preferable to those containing sugars

B Parents and carers should be advised that cheese is a good high energy food for toddlers as it is noncariogenic and may be actively protective against caries

GPP Parents and carers should be assured that sugar-free snacks are unlikely to be cariogenic

\section{Practice-based management}

D Primary teeth with caries progressing into dentine should be actively managed with a preventive, or a preventive and restorative, approach as appropriate with the child's ability to cooperate

B If complete caries removal from a vital primary molar is not possible an indirect pulp capping technique should be considered

B When preparing a Class II cavity, care must be taken to avoid iatrogenic damage to adjacent proximal tooth surfaces

B Use of the ART (Atraumatic Restorative Treatment) approach for cavity preparation in carious primary teeth should be considered as an alternative, where appropriate, to conventional cavity preparation techniques

A Amalgam, composite, resin-modified glass-ionomers, compomer or preformed metal crowns should be used as restorative materials for Class II cavities in primary molars. Conventional glass-ionomer should be avoided, where possible, for Class II cavity restoration

Practice-based prevention

B The dental health team should deliver caries prevention strategies in conjunction with physical prevention techniques such as the use of fluoride

B Parents and their preschool children should receive oral health education from their dental team. This should include oral hygiene instruction, the appropriate use of fluoride toothpaste and the provision of fluoride agents such as toothpaste

B Topical fluoride varnish should be applied to the dentition at least twice yearly for preschool children assessed as being at increased risk of dental caries

\section{Community-based prevention}

A Community or home-based oral health promotion interventions should use fluoride containing agents such as fluoride toothpaste

A Community-based toothbrushing programmes should include toothpaste with a concentration of 1000 ppm fluoride

B Toothbrushing programmes should be undertaken in community-based settings such as nurseries, and with parents to create a supportive environment for oral health behaviour

D Oral health promotion programmes to reduce the risk of early childhood caries should be available for parents during pregnancy and continued postnatally

C Programmes for young children should be initiated before the age of 3 years. The oral health of young children should be promoted through multiple interventions and multisessional health promotion programmes for parents

C Teachers, community workers and lay or peer educators can be effective in delivering health promotion interventions and their role should be considered in the development of oral health promotion programmes

Professionals should ensure oral health messages are relevant and applicable to communities and lifestyles

Caries prevention measures should target 'at-risk' populations and individuals to reduce oral health inequalities

Fluoride supplements should only be prescribed by dental practitioners on an individual patient basis 


\section{Commentary}

Clinical guidelines have been defined as systematically developed statements to assist practitioner and patient decisions about appropriate healthcare for specific clinical circumstances ${ }^{2}$ in order to condense a large body of medical knowledge into a convenient, readily usable format. ${ }^{3}$ In clinical practice, they are particularly relevant for two main reasons: first, the clinical guideline can review all the available evidence and synthesise it, whereas a systematic review would focus on high-quality evidence often only from RCT; and second, the clinical guideline differs from decision analyses in that it relies more on qualitative reasoning and in emphasising a particular clinical context. ${ }^{4}$

As the guideline authors here have noted, this "is not intended to be construed or to serve as a standard of care", but is clear that the ultimate intention was to influence what clinicians do.

The condition targeted here is dental caries in preschool children. This clinical guideline was formulated by a large and multidisciplinary team including clinicians, public health specialists and consumers' groups and organisations. It was developed for use by clinicians in Scotland, a country with one of the highest dental caries rates in Europe (mean DMFT, 2.7). Thus, the implicit goal of this guideline is to reduce the DMFT index in Scottish preschool children, at least, at the d3 level (frank decay). Although the primary target users would be dental teams working community dental clinics, ideally it would also reach parents, carers and crèche staff.

The guideline cover areas including dental caries diagnosis; caries risk assessment; dental caries prevention; and dental caries management in preschool children. It also has a chapter with recommendations for the implementation and posterior auditing of the guideline. The last chapter contains useful information for parents, which can be converted into a leaflet, and a one-page summary of all recommendations appropriate for a clinical setting.

The main body of the guideline contains statements on the evidence level that support them and the recommendation grade. It is very easy to read because it written as short paragraphs in which the evidence is explained and graded, from $1++$ (evidence from high-quality systematic reviews or RCT) to 4 (expert opinion). These paragraphs are followed by a short and clear statement with the corresponding grade of recommendation, from $\mathrm{A}$ to $\mathrm{D}$, according to the evidence level. It also has "good practice points", which are statements that represent best practice, based on the clinical experience of the guideline development group.

The critical appraisal of a clinical guideline involves three key questions: $^{4}$

(1) Are the results of this guideline valid? (ie, was an explicit and sensible process used to identify, select and combine evidence?)

(2) What are the results? (ie, are practical, clinically important recommendations made?)

(3) Will the results help me care for my patients? (ie, are the recommendations applicable to my patients?)

As alluded above, a clinical guideline is constructed using all the available evidence, not just high-quality data such as that from RCT. The large number of references (181) indicates that the search for articles published in English was exhaustive. This guideline cited 23 systematic reviews or meta-analyses.

One slightly curious aspect is that the definition of caries is taken from the book by Kidd ${ }^{5}$ although, later, the guideline's authors note that dental caries can be prevented, something explicitly denied in Kidd's book and in two others references cited by the authors. ${ }^{6,7}$ These references highlight that strictly speaking the caries process cannot be prevented, only controlled to the extent that a visible carious lesion does not develop.

What is clear is that the status of the primary dentition can predict caries in permanent teeth ${ }^{8-10}$ and therefore most of the recommendations are either: to intervene early or prevent carious lesions; carry out caries risk assessments; or specific dietary recommendations. For example, recommendations include toothbrushing with fluoride toothpaste (even for children under 2 years old, with a smear size amount of fluoride) and oral health promotion programmes for parents and continued postnatal care - a recommendation based on evidence from nonanalytical studies (case reports or case series) that in fact represents evidence from clinical trials. ${ }^{11}$

Of the 33 recommendations in the guideline, six are supported by high-quality evidence, 14 by a grade-B levels of evidence, nine with grade $\mathrm{C}$, and four with grade $\mathrm{D}$. The latter represents evidence from nonanalytical studies, expert opinion or that extrapolated from other studies. Even with the extensive panel of experts who developed this guideline, expert opinion was rarely used to fill the gaps found in literature.

The principal aim of evidence-based healthcare is to develop methods that can assist practitioners to provide the most appropriate, compassionate and ethical care to the public. ${ }^{12}$ The large body of evidence distilled in this guideline indicates that preventive and management measures should begin as early as possible, indeed before birth. In addition, oral health promotion programmes should include not only parents but also teachers and community workers to be effective. The instructions and recommendations given are not sufficient if carried out in the clinical setting alone: the dentist will have to play an important role as a community educator. One specific intervention, the use of fluoride at home, should be universal, regardless of a child's risk of caries. Meanwhile, as regards use in the clinic, the dental professional should choose the form of topical fluoride according to specific risk.

For healthy teeth or suspicious surfaces, sealants can be satisfactorily applied to primary molars. The management of frank or active carious lesions should be focused on the removal of infected dentine and the effective sealing of the cavity. For the treatment of extensive tooth decay, the guideline mentions that formocresol therapy should be replaced, but it does not give any alternative. This recommendation could have been strengthened if an alternative intervention had been suggested.

This guideline does not include a clinical algorithm, but its last page serves as a summary of recommendations. This is important because continued reinforcement of the recommendations should increase the impact of the guideline. ${ }^{13}$

Practitioners wish to use resources efficiently. This desire is met by the guideline because the majority of the recommended interventions are easy to implement in public dental settings. It is worth mentioning, however, that there is no recommendation about when to refer to a paediatric dentist.

Although this guideline was developed for use in Scotland, its recommendations can be implemented in any country that wishes to reduce the cost and pain associated with management of dental decay in preschool children. It is a call not to delay the prevention and treatment of dental caries to permanent teeth only. Fortunately, the times when primary teeth were seen as "replaceable" are over.

\section{Sergio Uribe \\ Department of Preventive and Paediatric Dentistry, University of Valparaiso, Valparaiso, Chile}

1. US Department of Health and Human Services. Acute Pain Management: Operative or Medical Procedures and Trauma. Rockville: Agency for Health Care Policy and Research, Clinical Practice Guideline No. 1. AHCPR Publication No. 92-0023; 1993, p 107.

2. Lohr KN, Field MJ. A provisional instrument for assessing clinical practice guidelines. In Guidelines for Clinical Practice. From Development to Use. Edited by Field M], Lohr KN. Washington, DC: National Academy Press; 1992.

3. Eddy DM. The Challenge. J Am Med Assoc 1990; 263:287-290.

4. Hayward RSA, Wilson MC, Tunis SR, et al. Users' guides to the medical literature. VIII. How to use clinical practice guidelines. A. Are the recommendations valid? J Am Med Assoc 1995; 274:570-574. 
5. Kidd EAM. Essentials of Dental Caries. 3rd Edn. Oxford: Oxford University Press; 2005; 2-7.

6. Featherstone JD. The continuum of dental caries - evidence for a dynamic disease process. J Dent Res 2004; 83(Suppl C):S39-S42.

7. Kidd EA, Fejerskov O. What constitutes dental caries? Histopathology of carious enamel and dentin related to the action of cariogenic biofilms. J Dent Res 2004; 83(Suppl C):S35-S38.

8. Broadbent JM, Thomson WM, Williams SM. Does caries in primary teeth predict enamel defects in permanent teeth? A longitudinal study. J Dent Res 2005; 84:260-264

9. Lo EC, Zheng CG, King NM. Relationship between the presence of demarcated opacities and hypoplasia in permanent teeth and caries in their primary predecessors. Caries Res 2003; 37:456-461.

10. Li Y, Wang W. Predicting caries in permanent teeth from caries in primary teeth: an eight-year cohort study. I Dent Res 2002; 81:561-566.
11. Gomez SS, Weber AA. Effectiveness of a caries preventive program in pregnant women and new mothers on their offspring. Int J Paediatr Dent 2001 11:117-122.

12. Ismail Al. Implications of evidence based health care for education. In Evidence-based Dentistry for Effective Practice. 1st edn. Edited by Clarkson I, Harrison JE, Ismail Al, Needleman I, Worthington H. London: Martin Dunitz; 2003; 166.

13. Weingarten SR, Riedinger MS, Conner $\mathrm{L}$, et al. Practice guidelines and reminders to reduce duration of hospital stay for patients with chest pain. An interventional trial. Ann Intern Med 1994; 120:257-263.

Evidence-Based Dentistry (2006) 7, 4-7.

doi:10.1038/sj.ebd.6400385 